\title{
Effects of Wrist Taping Pressures on the Maximum Dorsal Flexion Angle
}

\author{
Kenji Takahashi ${ }^{1, *}$, Shin-ichi Demura ${ }^{2}$ \\ ${ }^{1}$ Faculty of Community Health Care, Department of Judo Physical Therapy, Teikyo Heisei University, Uruidominami 4-1 Ichihara, \\ chiba, Japan \\ ${ }^{2}$ Graduate School of Natural Science \& Technology, Kanazawa University, Kakuma, Kanazawa, Ishikawa, Japan \\ *Corresponding author: kenji.takahashi@thu.ac.jp
}

Received April 16, 2014; Revised May 14, 2014; Accepted May 29, 2014

\begin{abstract}
This study aimed to examine the effects of different taping pressures with or without external force on the maximum dorsal flexion angle (DFA). Twenty-two healthy male university students with $>5$ years of athletic experience participated. A qualified trainer wound a rigid tape thrice around subject's wrist joint. Taping pressure was measured using the pressure measuring system AMI3037-SB. Independent variables were five experimental conditions (5 hPa, $30 \mathrm{hPa}, 60 \mathrm{hPa}$, and $90 \mathrm{hPa}$, and control) and two measurement conditions for the wrist joint DFA with non-external and external forces. In the former, the subject flexed the wrist joint himself (active DFA), while in the latter, a trainer helped in flexion (passive DFA). The dependent variable was the maximum DFA. Two-way repeated measures ANOVA (taping pressure $\times$ different DFA measurement) and multiple comparison tests showed that passive DFA was significantly larger than active DFA under all taping pressure conditions. Active DFA was smaller under 30-hPa condition than under the 5-hPa condition, and was smaller under 60-hPa conditions than under control and 5-hPa conditions, and was smaller under 90-hPa condition than under the other conditions. Passive DFA was smaller under 30- and 60-hPa conditions than under control and 5-hPa conditions, and was smaller under 90hPa condition than under control, 5-, and 30-hPa conditions. In conclusion, the passive DFA is larger than the active DFA, regardless of taping pressure. Wrist taping with pressure over $30 \mathrm{hPa}$ limits DFA, particularly at $90 \mathrm{hPa}$.
\end{abstract}

Keywords: pressure measuring system, rigid tape, blood flow, competitive sports, wrist joint

Cite This Article: Kenji Takahashi, and Shin-ichi Demura, "Effects of Wrist Taping Pressures on the Maximum Dorsal Flexion Angle.” American Journal of Sports Science and Medicine, vol. 2, no. 4 (2014): 143147. doi: 10.12691/ajssm-2-4-5.

\section{Introduction}

Athletes such as sumo and other wrestlers, judo fighter, weightlifters, and gymnasts tape their wrists with the aim of preventing wrist disorders or injuries by limiting excessive motion of the wrist joints [1,2]. Many injuries occur to the wrist joints because of excessive dorsal flexion [3,4]. Therefore, taping, which limits dorsal flexion, may be effective in preventing disorders or injuries. There are various methods for taping the wrist joint. Constantinou and Brown [1] introduced the following two methods: taping around the wrist joint (Figure1-a. wrist taping) and X support taping using crossbridge tape on the palmar surface (Figure 1-b. X taping).

Wrist taping has been primarily used to decrease load stress and prevent excessive dorsal flexion; however, the main purpose is the prevention of injuries [2]. Athletes have generally used this taping method because they can wind the tape themselves. This method slightly limits all movements of the wrist joint. However, the degree of limitation of the range of motion (ROM) has not been shown with an objective measurement value [1].
On the other hand, the $\mathrm{X}$ taping method is used instead of a brace after a wrist injury [1,2]. Yamamoto [5] reported that $\mathrm{X}$ taping limited an average $25^{\circ}$ of the maximum dorsal flexion angle (DFA) in American football players. This taping method is considered to have a strong fixing force because it involves taping of the palmar surface. However, this method may have disadvantages. For example, the palmar response may become insensitive and slippery when the individual grips objects. Therefore, in competitive situations, wrist taping should be preferred over $\mathrm{X}$ taping. The fixing force of wrist taping is weaker than that of $\mathrm{X}$ taping. However, $\mathrm{a}$ fixing force similar to that used in $\mathrm{X}$ taping may be obtained by increasing taping pressure.

Constantinou and Brown [1] pointed out the need to control taping pressure when examining the effects of taping in a review of other studies. It is considered that differences in taping pressure affect various factors such as strength exertion and ROM. For example, strong pressure may decrease blood circulation and cause nerve compression [6], because a tape wound around the wrist joint can excessively limit ROM. Therefore, it is necessary to examine the effects of different taping pressures on ROM.

In clinical practice, passive ROM is generally used to evaluate ROM of patients after injuries or surgeries [7]. 
Active ROM is also measured together with passive ROM during ROM evaluation of patients with muscle paralysis. In competitive sports, although athletes bend the wrist joints voluntarily, there are cases in which dorsal flexion is forced when the athlete receives some external force. Therefore, it is necessary to examine the effects of the passive DFA and the active DFA. Passive ROM is generally greater than the active ROM in all joints [7].

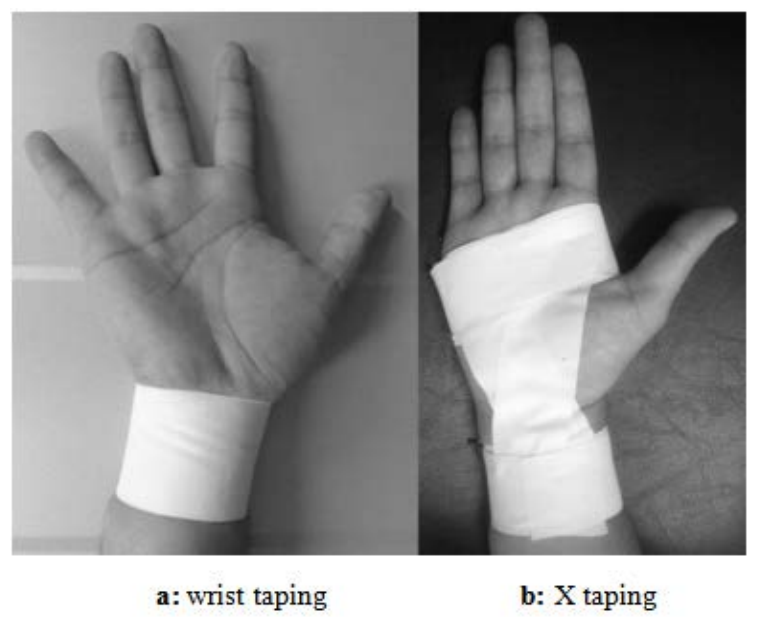

Figure 1. Taping method of wrist joint

Therefore, we hypothesized that limitation effects on DFA become greater with increasing taping pressure because of the increased physical load on the wrist joint, and that the DFA is greater in the passive ROM measurement than in the active one, even if taping pressure is imposed on the wrist joint. This study aimed to examine the effects of different taping pressures on the passive and the active DFA.

From the present study, we may be able to judge the degree of taping pressure to decide a limited range of motion required when performing the wrist joint taping in the competitive situation.

\section{Method}

\subsection{Subjects}

Participants were 22 male university students (mean age, $19.4 \pm 1.0$ years) with $>5$ years of athletic experience and no history of wrist injuries such as sprain, fracture, or dislocation. Their sports were as follows: baseball $(n=8)$, soccer $(n=4)$, basketball $(n=4)$, track and field $(n=1)$, judo $(n=1)$, kendo $(n=1)$, karate $(n=1)$, rugby football $(n=1)$, and swimming $(n=1)$. Figure 1 shows the basic statistics [age, height, body weight, body mass index (BMI), wrist circumference, and competitive sports experience] of the subjects.

The aim and procedures of this study were explained in detail to all subjects prior to the experiment, and informed consent was obtained. This experimental protocol was approved by the Ethics Committee on Human Experimentation of the Faculty of Human Science, Kanazawa University (2012-18).

Table 1. The basic statistics for age, height, body weight, BMI and circumference of the dominant wrist joint of subjects (n=22)

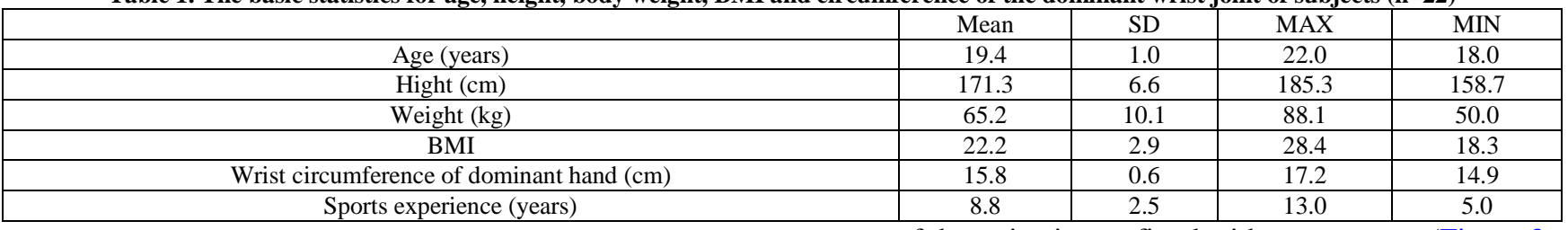

\subsection{Tape and Taping Method}

In this study, we used 50-mm rigid (non-elastic) tape manufactured by Johnson \& Johnson (New Brunswick, NJ, USA). We adopted the wrist-taping method, in which one piece of rigid tape is wound thrice around the wrist joint, including the radius and ulnar styloid processes (Figure 1a). An athletic trainer (Japan Sport Association) with $>10$ years of clinical experience performed the taping.

\subsection{Pressure-Measuring Device}

Taping pressure was measured using the pressure measuring system for stockings and bandages (AMI3037SB, AMI-Techno. Tokyo, Japan.) (Figure 2). This system can measure pressure on the human body through clothing such as socks [8] and can detect the addition of constant pressure such as that from the use of an elastic bandage [9]. Therefore, this device is considered to be useful in measuring taping pressure. The measurement unit was hPa. A measurable range was $1-200 \mathrm{hPa}$, and the measurement error was $\pm 3 \mathrm{hPa}$.

Taping was performed at the wrist joint of a participant's dominant hand. The dominant hand was judged according to Demura et al.'s handedness inquiry [10]. After a pressure sensor was placed on the palmaris longus muscle tendon $1.5 \mathrm{~cm}$ proximal to the palmar crease of the wrist, it was fixed with a cover tape (Figure 3 $\mathrm{a}, \mathrm{b}$, and c).

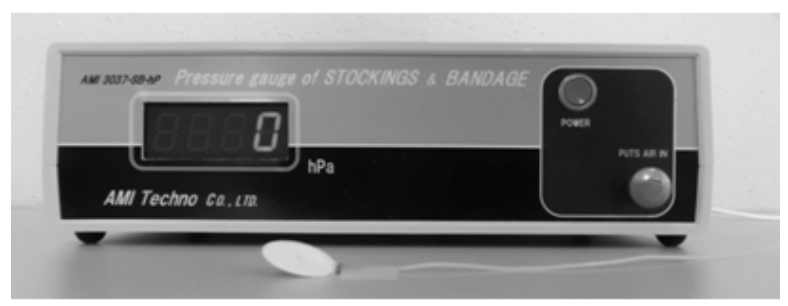

Figure 2. Pressure measuring system for stockings and bandages (AMI3037-SB)

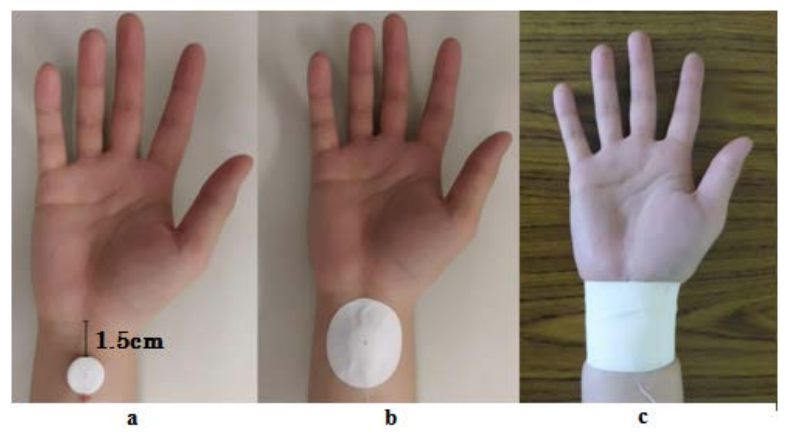

Figure 3. a: sensor only; b: sensor with cover tape; c: wrist joint taping with sensor \& cover tape 


\subsection{Independent and Dependent Variables}

The independent variables were five experimental conditions (pressure loads on the wrist joint), namely 4 pressure conditions of $5 \mathrm{hPa}, 30 \mathrm{hPa}, 60 \mathrm{hPa}$, and $90 \mathrm{hPa}$ and a control condition (no tape) without tape attachment, and two measurement conditions of external force (passive DFA) applied by a trainer and no external force (active DFA). Under the 5-hPa condition, the tape was only wound around the wrist without any pressure. We assumed that these experimental conditions would reveal the effects of different taping pressures on DFA of the wrist joint. The 5-hPa condition was adjusted within an

error range of $\pm 1 \mathrm{hPa}$, whereas the other pressures were adjusted within a range of $\pm 2 \mathrm{hPa}$. Dorsal flexion was measured under the following two conditions: under no external force, that is, when the subject flexed his own wrist joint maximally (active DFA), and under external force, when a qualified athletic trainer helped in flexion (passive DFA).

The dependent variable was the maximum DFA. The measurement unit was degree $\left({ }^{\circ}\right)$. A mean of three trials involving active and passive DFA measurements was used as an evaluation variable.

\subsection{Experimental Procedure}

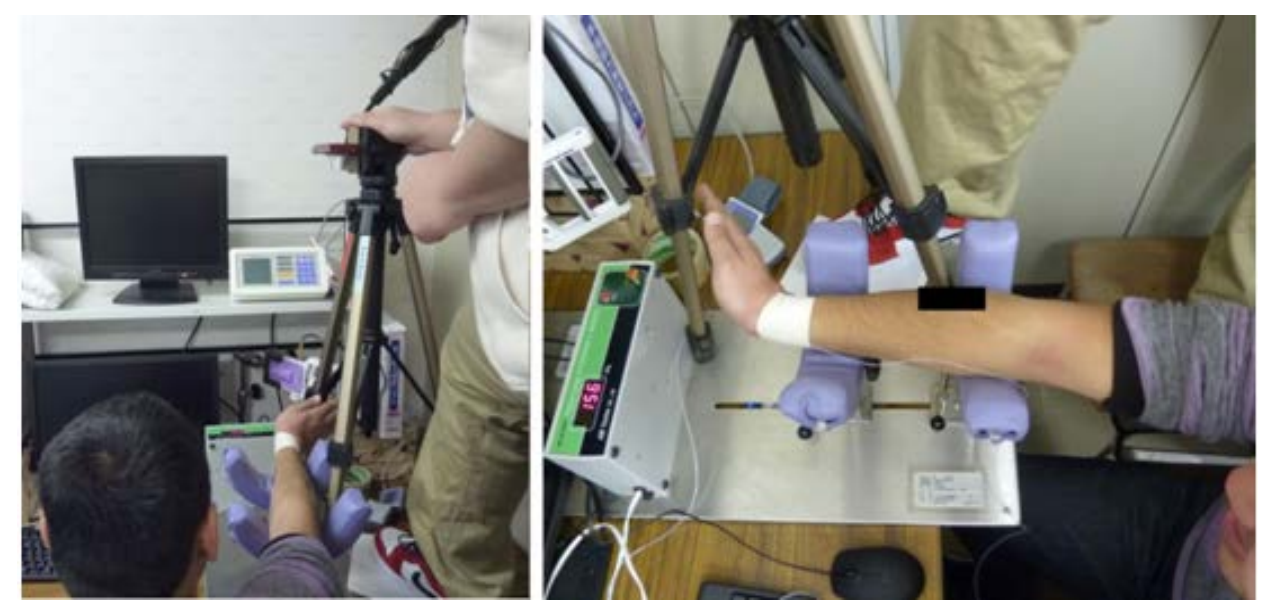

Figure 4. Measurement of (active) dorsal flexion angle using a digital camera with a leveler installed

Before the experiment, the subjects performed wrist warm-up exercises to prevent injury. Then, they assumed the following sitting posture: shoulder joint in 70-90 forward flexion, elbow joint in $15-30^{\circ}$ flexion, forearm in the middle position between pronation and supination, and wrist joint in the neutral position. The metacarpophalangeal joint, the proximal interphalangeal joint, and the distal interphalangeal joint were kept in the extension state. Using a digital camera (DSC-W730/P, SONY, Tokyo, Japan) with a leveler installed, images of the measurements were acquired from directly above (Figure 4). Setting the stationary arm as the radius and the movable arm as the second metacarpal, we calculated DFA of the wrist joint using a posture evaluation program Ver. 1.0.1. (Takei, Tokyo, Japan) on the basis of the measurement images. Pressure experimental conditions were randomized for every subject, and active and passive DFAs were measured thrice in each condition without a rest time between trials. A rest time between conditions was $>3$ min. Passive and active DFA measurements were conducted alternately to eliminate any order effects. The tape was replaced in each pressure condition. The experiment was performed from 9:00 $\mathrm{h}$ to $13: 00 \mathrm{~h}$ in a laboratory maintained at $26^{\circ} \mathrm{C}$.

\subsection{Statistical Analysis}

Two-way repeated measures ANOVA (taping pressure $\times$ different DFA measurement) was used to reveal the mean differences among each condition using SPSS 18 (SPSS Inc., Chicago, IL). When significant effects were found, we performed a multiple comparison test using the Tukey's HSD method. The level of significance was set a priori to 0.05 . The statistical power $(1-\beta)$ of interaction and main effect obtained in ANOVA was calculated in post-hoc analysis using G*Power 3.1.7 software (http://www.psycho.uniduesseldorf.de/abteilungen/aap/gpower3/) [12]. In addition, an effect size (ES) was calculated to examine the size of mean differences. ES was interpreted as follows: $<0.2$ as small, $>0.5$ as intermediate, and $>0.8$ as large.

\section{Results}

Table 2 shows the means and standard deviations of the active and passive DFAs under all experimental conditions. Results of the two-way repeated measures ANOVA (taping pressure $\times$ different DFA measurement) and the multiple comparison tests are also shown along with ES values. No significant difference was found in interaction ( $\mathrm{df}=4, \mathrm{~F}=2.19, \mathrm{p}=0.08$, partial $\eta 2=0.09,1$ $-\beta=0.98$ ), and significance was found in both main factors of the measurement method $(\mathrm{df}=1, \mathrm{~F}=328.78 \mathrm{p}<$ 0.05 , partial $\eta 2=0.94,1-\beta=1$ ) and taping pressure (df $=4, \mathrm{~F}=41.47, \mathrm{p}<0.05$, partial $\eta 2=0.66,1-\beta=1$ ). Multiple comparison tests showed that the passive DFA was larger than the active DFA under all experimental conditions [ES (d) $=4.22-5.28]$. Active DFA was smaller under the 30-hPa condition than under the 5-hPa condition [ES (d) $=0.58]$, and was smaller under the $60-\mathrm{hPa}$ condition than under control and 5-hPa conditions [ES (d) $=0.50,0.70]$, and was smaller under the $90-\mathrm{hPa}$ condition than under other conditions [ES (d) $=0.51-1.14$ ]. Passive DFA was smaller under 30 - and $60-\mathrm{hPa}$ conditions than under control and 5-hPa conditions [ES (d) $=0.73-1.58$ ], and was smaller under the $90-\mathrm{hPa}$ condition than under control, 5-hPa, and 30-hPa conditions [ES (d) = 0.66-1.93]. 
Table 2. Two-way repeated measures ANOVA (N=22)

\begin{tabular}{|c|c|c|c|c|c|c|c|c|c|c|c|}
\hline & & \multicolumn{4}{|c|}{ Dorsal flexion } & & \multirow{2}{*}{\multicolumn{3}{|c|}{ Two-way ANOVA }} & \multirow{3}{*}{ Tukey’s HSD } & \multirow{3}{*}{ Effect size (d) } \\
\hline & & \multicolumn{2}{|c|}{ Active DFA } & \multicolumn{2}{|c|}{ Passive DFA } & & & & & & \\
\hline & & Mean & SD & Mean & SD & & F-value & p-value & $\eta 2$ & & \\
\hline \multirow[t]{5}{*}{$\begin{array}{l}\text { Taping } \\
\text { pressure }\end{array}$} & Control & 63.30 & 7.85 & 97.91 & 4.92 & $\mathrm{~F} 1$ & $328.78 *$ & & & $\begin{array}{l}\text { Active DFA: } \\
\text { Con }>60,90 \\
\text { 5>30, } 60,>90 \text {. }\end{array}$ & $\begin{array}{l}0.50,0.96 \\
0.51-1.14\end{array}$ \\
\hline & $5 \mathrm{hPa}$ & 64.79 & 7.51 & 96.03 & 5.58 & & & & & Passive DFA: & \\
\hline & $30 \mathrm{hPa}$ & 60.18 & 8.29 & 91.91 & 5.79 & & & & & $\begin{aligned} \text { Con, } 5 & >30,60,90 \\
30 & <90\end{aligned}$ & $\begin{array}{c}0.73-1.93 \\
0.66\end{array}$ \\
\hline & $60 \mathrm{hPa}$ & 59.30 & 8.19 & 90.17 & 4.92 & $\mathrm{~F} 2$ & $41.47^{*}$ & 0.00 & 0.66 & \multirow{2}{*}{$\begin{array}{c}\text { Con, 5, 30, 60, 90: Passive } \\
\text { DFA>Active DFA }\end{array}$} & \multirow{2}{*}{$4.22-5.28$} \\
\hline & $90 \mathrm{hPa}$ & 54.65 & 10.11 & 88.32 & 5.01 & IN & 2.19 & 0.08 & 0.09 & & \\
\hline
\end{tabular}

$*: \mathrm{p}<0.05$

F1: Active DFA * Passive DFA F2: Taping pressure IN: interaction

Con: Control 5: 5hPa, 30: 30hPa, 60: 60hPa, 90: 90hPa

\section{Discussion}

Generally, ROM of the wrist joint becomes larger when an external force is added by the examiner than when individuals move the joint themselves. Norkin and White [7] reported that the margins protect the joint structure from external forces. Researchers have not considered the limiting effects of wrist-taping with taping pressure on ROM [1]. This study therefore clarifies that the passive DFA is larger than the active DFA, regardless of taping pressure magnitude. Therefore, DFA becomes larger under added external forces, even when the wrist is taped.

A taping pressure of $>30 \mathrm{hPa}$ limited both active and passive DFAs, whereas that of $5 \mathrm{hPa}$ did not. In particular, the limiting effects were stronger under the $90-\mathrm{hPa}$ condition than under the no-tape condition ( $\mathrm{ES}=0.96$, 1.93). Therefore, regardless of external force, it can be said that the limiting effect of DFA cannot be expected by wrist-taping without pressure; it can be expected under the application of pressures $>30 \mathrm{hPa}$, particularly $90 \mathrm{hPa}$.

The limiting effect probably occurred under the 30-hPa condition because the tape acts as a splint when a certain level of tension is applied. Conversely, no limiting effect was achieved under the 5-hPa condition, probably because the tension required for the tape to act as a splint was not achieved at a pressure of $5 \mathrm{hPa}$.

It is inferred that the strong limiting effect achieved under the $90-\mathrm{hPa}$ condition is due to the splint effect and taping pressure applied to the joint structure. Shimawaki et al. [11] reported that veins were constricted on addition of a pressure of $>60 \mathrm{mmHg}(80 \mathrm{hPa})$ to the upper arm. Therefore, a pressure of up to $60 \mathrm{hPa}$ was decreased by veins located just below the surface of the wrist joint; this pressure extends to the joint structure at $90 \mathrm{hPa}$. However, we believe that there was no difference in limiting effect between the 30 - and $60-\mathrm{hPa}$ conditions because only the splint effect was applied.

Under the 90-hPa (77.25 mmHg) condition in our study, we found a slight pressure change $(1-3 \mathrm{hPa})$ in all subjects, as observed by the pulse and a change in complexion of the hand. We thought that taping pressure close to the diastolic blood pressure at the wrist joint may cause a slight pressure change. Shimawaki et al. ${ }^{[11]}$ reported that blood volume in the finger increased gradually by increasing the pressure in increments, when applied with pressures of 20-80 mmHg over the upper arm. Moreover, a pressure close to the diastolic blood pressure causes constriction of veins and blood flow changes enough for individuals to feel their pulse. Therefore, in future, it will be necessary to examine blood flow change according to differences in taping pressure.

In this study, the procedure of attaching the tape, adjusting the pressure, and completing the measurements took approximately $4 \mathrm{~min}$. In any case, we avoided prolonged taping of the wrist with a pressure of $90 \mathrm{hPa}$. We did not find a slight pressure change under the $60-\mathrm{hPa}$ condition. However, in this condition, it may be possible to palpate the pulse because there is much individual variation in blood pressure. Regarding the problem of blood pressure and blood flow mentioned above, it would also be necessary to examine the $60-\mathrm{hPa}$ condition in addition to the $90-\mathrm{hPa}$ condition in relation to the duration of taping.

A change in the complexion of the hand was not found under the $30-\mathrm{hPa}$ condition (approximately $4 \mathrm{~min}$ ). The effect on the blood flow is small during wrist-taping with a pressure of $30 \mathrm{hPa}$. The limiting effect on dorsal flexion was not higher at the $60-\mathrm{hPa}$ condition than at the $30-\mathrm{hPa}$ condition. Therefore, if a taping pressure of $30 \mathrm{hPa}$ can result in the same limiting effect as that with $60-\mathrm{hPa}$, we recommend that there is no requirement to increase the taping pressure to $>30 \mathrm{hPa}$.

Constantinou and Brown [1] introduced some wrist joint taping methods such as wrist-taping and $\mathrm{X}$ support taping ( $\mathrm{X}$ taping). Yamamoto et al. [5] reported considerable limitation effects by $\mathrm{X}$ taping. As mentioned above, wrist-taping with a pressure of $>30 \mathrm{hPa}$ also limited DFA. In the future, it will be necessary to compare the limiting effects of $\mathrm{X}$ taping and wrist-taping in the same subjects. In addition, it will be necessary to compare the limiting effects of both taping methods and examine the effects on other joint movements such as palmar, ulnar, and radial flexion under the same experimental conditions.

In competitive situations, high-pressure wrist-taping is considered effective to prevent disorders and injuries by limiting the maximum DFA. However, prolonged taping may inhibit blood flow and result in decreased 
performance. Therefore, it is necessary to adjust taping pressure according to the sport and duration of the competition.

As practical applications to competitive sports, wristtaping with high pressure may not be adequately for athletes requiring consecutive strength exertion for a prolonged time, such as judo fighters, because they negatively affect their performance by the time to inhibition of blood flow becomes long. On the other hand, it will affect little performance of athletes such as weightlifters because the performance time is very short. In addition, when obtaining a limiting effect of dorsal flexion regardless of a difference of the athletic event, it may be necessary to use the taping pressure of $>30 \mathrm{hPa}$.

There are some methods of wrist-taping other than that used in this study [2]. In addition, elastic tape has been also used in the athletic field. Therefore, it is considered that the effect of taping pressure differs on the basis of the type of tape and taping method, and one limitation of study is that the results of this study apply only to the taping method using in this study (i.e., rigid tape). Future studies must examine the effects of range of motion caused by different types of tapes and taping methods.

\section{Conclution}

This study examined the effects of different taping pressures on the maximum DFA using the wrist-taping method, in which one piece of rigid tape is wound thrice around the wrist joint. Regardless of the magnitude of taping pressure, the passive DFA is larger than the active DFA. In both active and passive DFA measurements, the limiting effects on DFA can be expected from a taping pressure of $>30 \mathrm{hPa}$, particularly $90 \mathrm{hPa}$. However, a pressure of $>90 \mathrm{hPa}$ raises concerns about the negative effects of constricted blood flow. It is therefore necessary to adjust taping pressure according to the duration that the tape will be worn and the characteristics of the athlete and the sport.

\section{References}

[1] Constantinou, M., Brown, M., "Therapeutic taping for musculoskeletal conditions.” Elsevier: Churchill Livingstone, 2010.

[2] Rose, M., "Pocketbook of Taping Techniques.” Elsevier, Churchill Livingstone 2010.

[3] Linscheid, RL., Dobyns, JH., "Athletic injuries of the wrist.” Clin Orthop 198:141-151, 1985.

[4] Thomas, S., "Wrist Injuries: Part I.” Dynamic Chiropractic Feb 10, Vol. 15, Issue 04, 1997

[5] Yamamoto, I., Ichimiya, S., Kibamoto, K., Morita, H., Nakajima, T., Suzuki, A., "Athletic taping for preventing injury was performed for healthy athletes before exercise" [in Japanese]. Bulletin of NSSU 15 (2): 71-77, 1986.

[6] Hirata, K., Yoshida, M., "Effect of cuff compression intensity and cuff width on Skin blood flow measured by a laser-Doppler flowmeter” [in Japanese]. J JRA for Textile End-Use 36: 154-161, 1995.

[7] Norkin, CC., White, DJ., "Measurement of Joint Motion: Guide to Goniometry (4 edition ).” F.A. Davis Company 2009.

[8] Ooizumi, Y., Matsuzawa, E., Iida, Kenichi., "Establishment of evaluation methods of clothing pressure of high supported clothesThe relation between clothing pressure of stretch clothes measured on a dummy and the human body” [in Japanese]. Bulletin of TIRI 2: 120-121, 2007.

[9] Hirai, M., "Clinical application of elastic stockings” [in Japanese]. Jpn J Phle 18 (5): 239-245, 2007.

[10] Demura, S., Sato, S., Nagasawa, Y., "Re-ex-amination of useful items for determining hand domi-nance” [in Japanese]. Arch Sci Med (Torino) 168: 169-177, 2009.

[11] Shimawaki, S., Sakai, N., Tamura, H., "Change in Blood Vessel Images in Human Finger Using Near-Infrared Radiation while Compressing the Upper Arm: Effect of the Compression Pressure and the Age of Subjects" [in Japanese]. Transactions of the Jpn Society of Mechanical Engineers, Part C 75 (756): 2280-2285, 2009.

[12] G*power 3.1.7 software. Heinrich Heine University Düsseldorf. (http://www.psycho.uni-duesseldorf.de/abteilungen/aap/gpower3/). 